\title{
Unexpected Magnetism of Small Silver Clusters
}

\author{
M. Pereiro, ${ }^{1,2}$, D. Baldomir, ${ }^{1,2}$ and J. E. Arias $^{2}$ \\ ${ }^{1}$ Departamento de Física Aplicada, Universidade de Santiago de Compostela, Santiago de Compostela E-15782, Spain. \\ ${ }^{2}$ Instituto de Investigacións Tecnolóxicas, Universidade de Santiago de Compostela, Santiago de Compostela E-15782, Spain.
} (Dated: September 3, 2018)

\begin{abstract}
The ground-state electronic, structural, and magnetic properties of small silver clusters, $\mathrm{Ag}_{n}$ $(2 \leq \mathrm{n} \leq 22)$, have been studied using a linear combination of atomic Gaussian-type orbitals within the density functional theory. The results show that the silver atoms, which are diamagnetic in bulk environment, can be magnetic when they are grouped together in clusters. The $\mathrm{Ag}_{13}$ cluster with icosahedral symmetry has the highest magnetic moment per atom among the studied silver clusters. The cluster symmetry and the reduced coordination number specific of small clusters reveal as a fundamental factor for the onset of the magnetism.
\end{abstract}

PACS numbers: 36.40.Cg,36.40.Qv,31.15.Ar

In the last two decades, the research field of clusters has shown a rapid development in both experimental and theoretical investigations [1], since the clusters are well suited for several applications. For example, there has been a traditional interest in applications to catalysis [2], due to the considerable surface/volume ratio of clusters. More recently, clusters or nanoparticles that possess magnetic properties have offered exciting new opportunities for biomedical applications including (i) magnetic separation of labeled cells; (ii) therapeutic drug delivery; (iii) hyperthermic treatment for malignant cells; (iv) contrast enhancement agents for magnetic resonance imaging applications; (v) and also very recently for manipulating cell membranes [3].

Clusters are on the border line between atoms and bulk and thereby, they play an important role in understanding the transition from the microscopic structure to the macroscopic structure of matter. Although $4 d$ and $5 d$ transition metal atoms have unfilled localized $d$ states, none of them are magnetic. Only a few of the $3 d$ transition metals form magnetic solids. Thus, from the magnetic point of view, one of the long standing problems in condensed matter physics is to understand why some nonmagnetic metals become magnetic when they condense into clusters. There are two factors characteristic of clusters that mainly contribute to the onset and enhancement of the magnetism, namely, the reduced coordination number and the high symmetry since that, symmetry enables degeneracy and degeneracy spawns magnetism [4]. According to this, an icosahedral structure is a good candidate for the appearance of the magnetism because the maximal degeneracy of an irreducible representation of the icosahedra $\left(\mathrm{I}_{h}\right)$ group is 5 whereas all other cluster symmetries allow at most three-fold degeneracy, as was reported by Reddy et al. for $\mathrm{Pd}, \mathrm{Rh}$, and $\mathrm{Ru}[5]$.

In this letter, we present first-principles calculations on small silver clusters exhibiting an important magnetism,

*Email address: fampl@usc.es which has not been predicted until now. In the present work, we study the evolution of the magnetism of silver clusters as a function of the cluster size and special emphasis is placed on the effects of cluster symmetry. The magnetic behavior that we got is very interesting, if not astonishing, compared with the silver bulk magnetic properties. To the best knowledge of us, nobody has predicted or even observed magnetism in small bare silver clusters, however our computational simulations anticipate the onset and enhancement of the magnetism for the silver clusters that gather special conditions like high symmetry, and reduced coordination number. In this respect, the $\mathrm{Ag}_{13}$ cluster exhibits the highest magnetic moment among the studied clusters and converges into a very stable structure.

With the aim of studying the magnetic properties of small silver clusters, $\mathrm{Ag}_{n}(2 \leq \mathrm{n} \leq 22)$, we have performed density functional theory-based calculations consisting of a linear combination of Gaussian-type-orbitals KohnSham density-functional methodology as implemented in the DEMON-KS3P5 program package [6]. All-electron spin-unrestricted calculations were carried out at the generalized gradient approximation (GGA) level to take the exchange-correlation (XC) effects into account 7]. Localdensity approximation sometimes yields inaccurate bond lengths and total energies due to the insufficiency in describing the strong correlation effect of the localized $d$ electrons and charge density inhomogeneities. In these regards, the GGA should be a better choice [8]. For this reason, at the beginning of this work and to satisfy ourselves that the numerical procedure is reliable, we initiate a search of the functional that better fitted the calculated bond length of the silver dimer to the experimental one. We found that the functional that better fitted the bond length was the one developed by Perdew and Wang 7], given a bond length of $2.535 \AA$, that is in excellent agreement with the experimental measure $(2.53350 \AA)$ reported in Ref. [9]. An orbital basis set of contraction pattern $\left(633321 / 53211^{*} / 531+\right)$ was used in conjunction with the corresponding $(5,5 ; 5,5)$ auxiliary basis set for describing the $s, p$ and $d$ orbitals [10]. The grid for numerical evaluation of the XC terms had 128 radial shells 


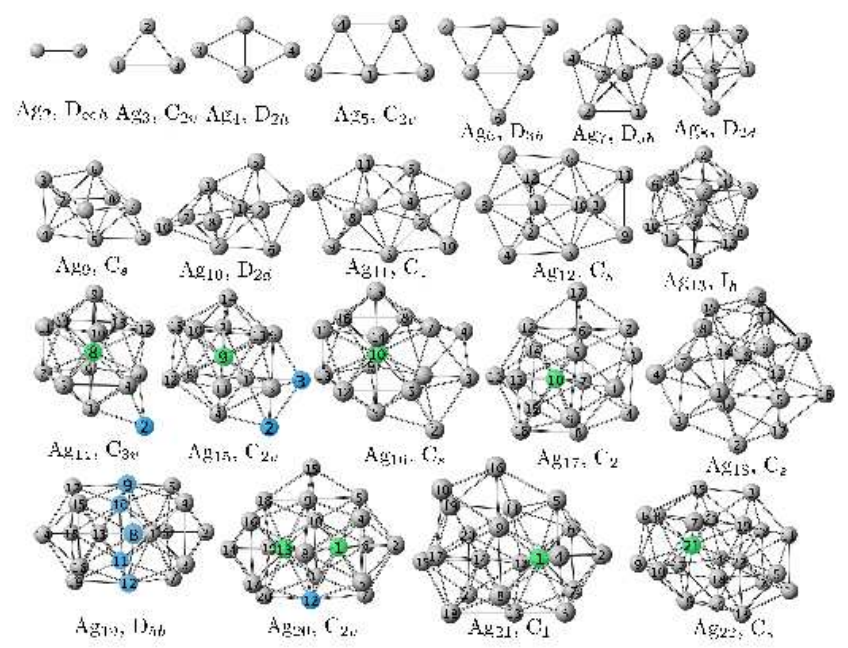

FIG. 1: (Color online). Lowest energy structures and the symmetry point groups assigned to the $\operatorname{Ag}_{n}(2 \leq \mathrm{n} \leq 22)$ clusters. The magnetic moments, namely $\mathrm{m}$, of the atoms showed in green $\left(-1.5 \mu_{B} \leq \mathrm{m} \leq-0.4 \mu_{B}\right)$ and blue $\left(-0.1 \mu_{B} \leq \mathrm{m} \leq 0 \mu_{B}\right)$ are aligned antiferromagnetically to the other ones.

of points and each shell had 26 angular points. Spurious one-center contributions to the XC forces, typically found in systems with metal-metal bonds when using a nonlocal functional, are eliminated in a similar way as has been done in Ref. 11]. A wide set of spin multiplicities ranging from 1 to 11 was checked to ensure that the lowest-energy electronic and magnetic configuration is reached. The geometries were fully optimized without symmetry and geometry constraints using the BroydenFletcher-Goldfarb-Shanno algorithm [12]. During the optimization, the convergence criterion for the norm of the energy gradient was fixed to $10^{-4}$ a.u. while it was $10^{-7}$ a.u. for the energy and $10^{-6}$ a.u. for the charge density. A huge sampling of trial geometries taken from the literature was evaluated. While for these small clusters it is nearly impossible to search for all possible geometries, the detailed search that we have carried out give us some confidence that the structural minima has been found. For clusters with size varying from 2 up to 12, the structural minima resulting from our calculations are closely related to the ones reported by R. Fournier [13], whereas all other cluster structures are quite similar to the ones of Ref. 14]. In the early stage of the geometry optimization process for silver clusters with size ranging from 13 to 22 and with the aim of speeding up the calculations, the structure of the silver clusters was first optimized in conjunction with a 17-electron scalar relativistic model core potential designed for the adequate description of the silver dimer bond length [15]. Once the geometry of the cluster was converged for the model core potential, an all-electron structural-relaxation calculation was performed leading to the current lowest energy structures showed in Fig. 1

One of the criteria for a cluster to be used as a potential building block for a nanomaterial is its chemical stability relative to other clusters of the same material. With the aim of studying the stability of $\mathrm{Ag}_{2}-\mathrm{Ag}_{22}$ clusters, we have plotted the HOMO-LUMO gaps, and the cohesive energies in Fig. 2(a),(b). The cohesive energies for $\mathrm{Ag}$ clusters are fitted to a linear regression and compared in Fig. 2(b) with the spherical droplet model of Miedema [16]

$$
E_{c}(n)=E_{b c}-\left(\frac{36 \pi}{n}\right)^{1 / 3} \gamma^{0} V_{a}^{2 / 3},
$$

where $\mathrm{E}_{b c}$ is the bulk cohesive energy, $\gamma^{0}=7.8 \times$ $10^{18} \mathrm{eV} / \mathrm{m}^{2}$ is the surface energy of the bulk silver, $\mathrm{V}_{a}$ is the atomic volume, and $\mathrm{n}$ is the number of atoms in the cluster. Despite that some clusters $(2 \leq \mathrm{n} \leq 6)$ are far from spherical, our calculated cohesive energies are in good agreement with the Miedema's model. Indeed, the bulk cohesive energy predicted by our calculations $(2.63$ $\mathrm{eV} /$ atom) differs by only $10 \%$ from the experimental findings (2.95 eV/atom) [17], given us confidence that the lowest energy structures plotted in Fig. [1 are reliable. In Fig 2(a) we can see that the most stable even-numbered clusters are $\mathrm{Ag}_{2}, \mathrm{Ag}_{6}, \mathrm{Ag}_{8}$ and $\mathrm{Ag}_{18}$. Except for $\mathrm{Ag}_{6}$, it is a consequence of the closure of the electronic shell [18]. For the odd-numbered clusters, the HOMO-LUMO gaps decrease monotonically as the cluster size increase, except for the $\mathrm{Ag}_{13}$ cluster. The large HOMO-LUMO gap of $\mathrm{Ag}_{13}$ relative to the odd-numbered clusters enhances its chemical stability and inertness, as well as its ability to assemble into magnetic nanoparticles because it is a magnetic cluster with a considerable magnetic moment per atom, as is discussed below.

Our results on the magnetic properties of the $\mathrm{Ag}_{n}$ $(2 \leq \mathrm{n} \leq 22)$ clusters are most fascinating, if not unexpected, compared with the silver bulk magnetic properties. Indeed, the silver bulk magnetic ordering is well-known to be diamagnetic, as can be inferred from its negative magnetic susceptibility $\left(\chi_{m}=-19.5 \times 10^{-6}\right.$ $\mathrm{cm}^{3} / \mathrm{mol}$ ), whereas silver atoms when they coagulate to a cluster become either magnetic or non-magnetic depending on the cluster size, as is shown in Fig. 3. For $\mathrm{Ag}_{2}$, $\mathrm{Ag}_{8}$ and $\mathrm{Ag}_{18}$ clusters, our calculations clearly show that they are highly stable with large HOMO-LUMO gaps [see Fig. 2(a)] and diamagnetic [see Fig. 3. The phenomenon can be understood simple from the 2,8 , and 18-electron rule, as described by the cluster shell models [19]. The former rule predicts transition metal clusters to be stable and diamagnetic when the valence shell of the metal atom contains 2, 8 or 18 electrons that completely fill electron shells forming closed electronic structures with paired spins.

From $\mathrm{Ag}_{3}$ up to $\mathrm{Ag}_{12}$ excluding $\mathrm{Ag}_{2}$ and $\mathrm{Ag}_{8}$, the cluster topologies converge into a geometry where all atoms belong to the surface [Fig. 1], and consequently with a reduced coordination number [Fig. B(b)]. This condition favors a reduced charge accumulation in nearest neighbor atoms that according to the calculated Mulliken population analysis (MPA) is less than 0.1 electron per atom in 

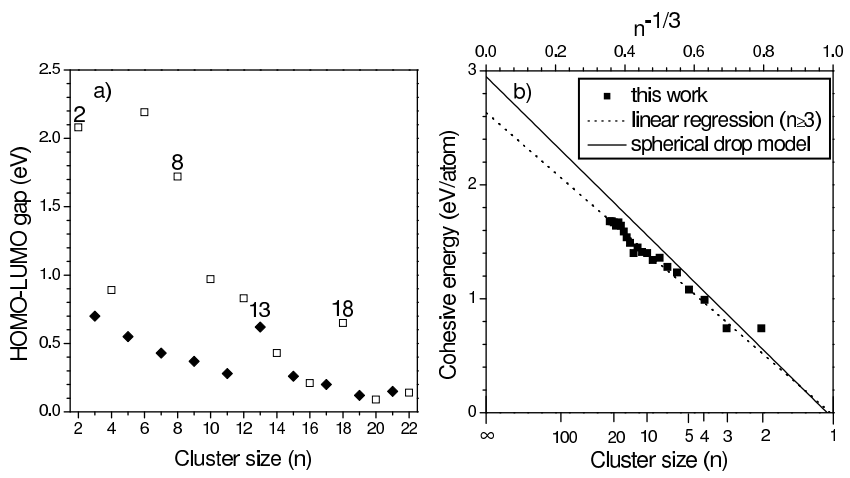

FIG. 2: (a) The HOMO-LUMO gap, and (b) the cohesive energy per atom of small silver clusters as a function of the cluster size $n(2 \leq n \leq 22)$.
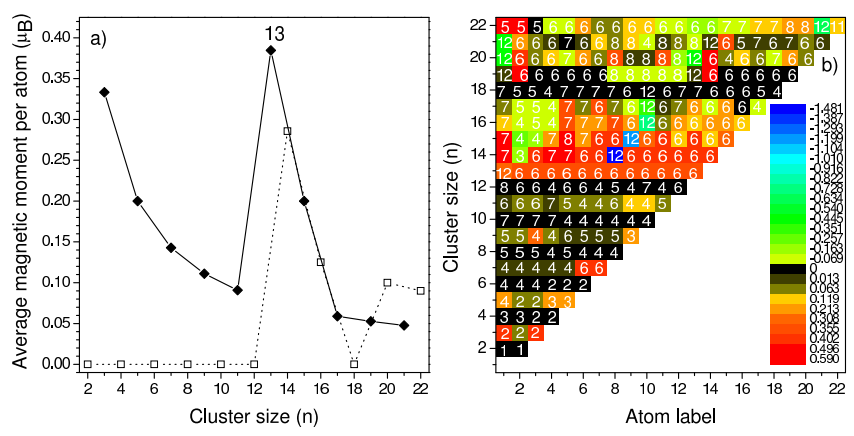

FIG. 3: (Color online). Evolution of magnetic moment per atom of the most stable cluster isomers of each size. (a) Average magnetic moment per atom versus cluster size. (b) Color representation of the magnetic moment per atom of each silver cluster. The numbers inside the small charts denote the coordination number. On the right side, the color palette gives information about the numerical values of the atomic magnetic moments. The numbers labeling every atom of each cluster in Fig. 1 are closely related to the ones displayed in $\mathrm{x}$-axis.

average. Likewise, the Mayer bond order analysis reveals that silver atoms are sharing the unpaired $5 s$ electrons forming a covalent bond and thereby, low spin configurations are expected. For example, the even-numbered clusters become diamagnetic because they have an even number of $5 \mathrm{~s}$ electrons that create a cloud of paired $5 \mathrm{~s}$ electrons [Fig. [3(a)], whereas for the odd-numbered clusters the electronic configuration with one unpaired spin electron is favored energetically over the whole feasible spin configurations studied in this work. The resulting odd-numbered clusters retain an average magnetic moment per atom that decreases monotonically with the increase of the coordination number [Fig. [3(a),(b)] since the orbital overlap expands as the coordination number does, such as was reported for Fe, Co and Ni in Ref. 20] and for 13-atom clusters of $\mathrm{Pd}, \mathrm{Rh}$, and $\mathrm{Ru}$ in Ref. [5].

In Fig. 31(a) we can see that $\mathrm{Ag}_{13}$ exhibits the highest average magnetic moment per atom among the studied clusters. To understand the origin of this considerable
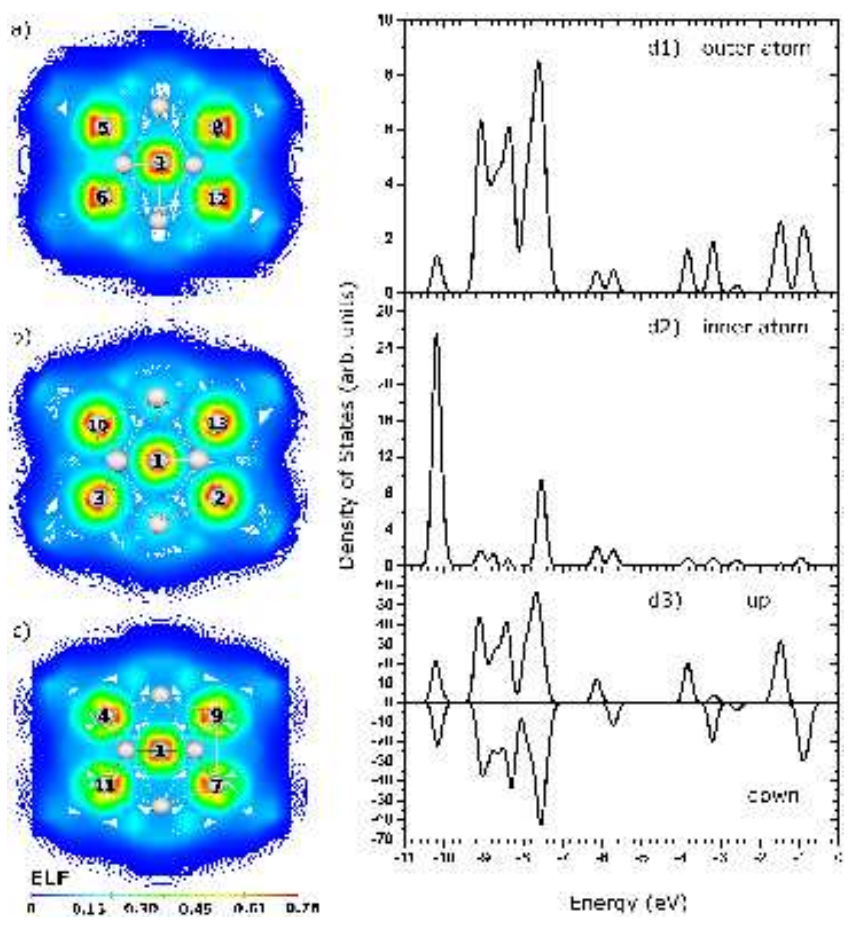

FIG. 4: (Color online). Contour plot of the electron localization function for the three mutually orthogonal golden planes ((a), (b), (c)) of the icosahedral symmetry and densities of states (DOS) for the outer-shell atoms (d1), and inner atom (d2) in an $\mathrm{Ag}_{13}$ cluster. The spin-polarized DOS is shown in (d3). The dashed vertical line represents the Fermi level.

magnetic moment $\left(0.39 \mu_{B}\right.$ /atom $)$ we show in Fig. 4l(a)(d3) the electron localization function (ELF) and the densities of states (DOS) for the inner and the outer-shell atoms. The MPA reveals that the electron charge transfers from the outer-shell Ag atoms to the central Ag atom which gains an excess of charge of about 0.8 electrons. The charge transfer, that is favored by the icosahedral symmetry, can be described graphically from our corresponding ELF plot. In Fig. [4(a)-(c), the contour plot of the ELF indicates a slight concentration of the light blue color (0.15-0.30 in bar color) surrounding the central atom, and give rise to an enhancement of the electron localization around inner atom. The large coordination number of the central $\mathrm{Ag}$ atom results in an enhancement of the overlap of its $4 d$ orbitals with those of other outershell Ag atoms. Therefore, for the inner atom the charge transfer populate mainly the lower energy $4 d$ states $(\sim$ $10.2 \mathrm{eV}$ ) with reduced exchange splitting whereas for the outer-shell atoms these states become less occupied, as is shown in Fig. 41(d1),(d2) 21] and Fig. 4(d3), respectively. Thereby, the charge transfer lead to a small loss of the spin-up DOS at the Fermi level for the inner atom compared with the outer-shell atoms and consequently, the inner atom weakens its magnetism $\left(0.35 \mu_{B}\right.$ /atom $)$ and the outer-shell atoms enhance their magnetic moments (0.39 $\mu_{B}$ /atom $)$ given rise to an increase of the average magnetic moment of the $\mathrm{Ag}_{13}$ cluster. 
From $\mathrm{Ag}_{14}$ to $\mathrm{Ag}_{22}$ except for $\mathrm{Ag}_{18}$ that have already been studied above, the clusters converge into a distorted icosahedral symmetry that depending on the cluster size, the number of inner atoms is either one or two (see Fig 1). In this case, the MPA confirms a charge transfer mainly from the peripheral atoms to the inner ones even greater than the $\mathrm{Ag}_{13}$ case. This effect decompensate the spin pairing state and give rise to an antiferromagnetic alignment of the inner atoms to the outer ones and thus, the clusters decrease the total magnetic moment compared with the $\mathrm{Ag}_{13}$ cluster, as is shown in Fig. 11 and Fig. 3(b). It is also observed in Fig. [3) a) that the average magnetic moment per atom decreases as the cluster size gets bigger up to $n=19$ and then, oscillates tending to decrease. The phenomenon can be understood simply from the loss of symmetry that reduces orbital degeneracy and weakens magnetism [4]. According to this tendency, we expect for greater clusters in size an enhancement of the magnetic moment as long as the cluster stabilizes in a geometry of high symmetry (e.g., $\mathrm{Ag}_{38}, \mathrm{Ag}_{55}$, and $\mathrm{Ag}_{75}$ ).

In conclusion, we have shown that the silver atoms can be magnetic when they are grouped together in small clusters. Particularly, the $\mathrm{Ag}_{13}$ cluster exhibits the highest magnetic moment per atom among the studied silver clusters due to its high symmetry and degeneration.

The authors acknowledge the CESGA (Centro de Supercomputación de Galicia), especially A. Gómez and C. Fernández for computational assistance. The work was supported by the Xunta de Galicia under the Project No. PGIDIT02TMT20601PR.
[1] H. Haverland, ed., Clusters of Atoms and Molecules: Theory, Experiment, and Clusters of Atoms. (Springer, Berlin, 1994).

[2] C. R. Henry, Surf. Sci. Rep. 31, 235 (1998).

[3] C. C. Berry and A. S. G. Curtis, J. Phys. D:Appl. Phys. 36, R198 (2003); Q. A. Pankhurst, J. Connolly, S. K. Jones, and J. Dobson, ibid. 36, R167 (2003); P. Tartaj et al., ibid. 36, R182 (2003).

[4] B. I. Dunlap, Phys. Rev. A 41, 5691 (1990).

[5] B. V. Reddy, S. N. Khanna, and B. I. Dunlap, Phys. Rev. Lett. 70, 3323 (1993).

[6] A. St-Amant and D. R. Salahub, Chem. Phys. Lett. 169, 387 (1990).

[7] J. P. Perdew and Y. Wang, Phys. Rev. B 46, 12947 (1992).

[8] M. Pereiro et al., Int. J. Quantum Chem. 81, 422 (2001).

[9] B. Simard et al., Chem. Phys. Lett. 186, 415 (1991).

[10] S. Huzinaga et al., Gaussian Basis Sets for Molecular Calculations (Elsevier, Amsterdam, 1984).

[11] L. Versluis and T. Ziegler, J. Chem. Phys. 88, 322 (1988).

[12] H. B. Schlegel, Modern Electronic Structure Theory
(World Scientific, Singapore, 1995), chap. 8, p. 459.

[13] R. Fournier, J. Chem. Phys. 115, 2165 (2001).

[14] The Cambridge Cluster Database, http://www-wales.ch.cam.ac.uk/CCD.html.

[15] E. R. J. Andzelm and D. R. Salahub, J. Chem. Phys. 83, 4573 (1985).

[16] A. R. Miedema, Z. Metallkd. 69, 287 (1978).

[17] C. Kittel, Introduction to Solid State Physics (Wiley, New York, 2005), 8th ed.

[18] M. Pereiro and D. Baldomir, Phys. Rev. A 72, 045201 (2005).

[19] R. H. Crabtree, The Organometallic Chemistry of the Transition Metals (Wiley, New York, 1988).

[20] F. Liu et al., Phys. Rev. B 39, 6914 (1989).

[21] In Fig. 4(d1), we show the DOS for the atom labeled with the number 2 in Fig. 1 as a representative example of the DOS for the outer-shell atoms, since from symmetry considerations the external atoms have all of them similar DOS plot. 